Research Article

\title{
Development of Prototype Microwave Radar-based Imaging System for Breast Cancer Detection
}

\author{
Humza Sami ${ }^{1}$, Mahnoor Sagheer ${ }^{1}$, Muhammad Amir Altaf $^{1}$, Javaid Iqbal $^{2}$ and Muhammad Zubair ${ }^{1, *}$
}

${ }^{1}$ Electrical Engineering Department, Information Technology University, Labore, Pakistan; ${ }^{2}$ Department of Mechatronics Engineering, National University of Sciences and Technology, H-12, Islamabad, Pakistan

\begin{abstract}
In this work, a complete microwave imaging system is designed and implemented for the reconstruction of breast tumor images. An UWB Vivaldi antenna is used in the bandwidth of 2-6 GHz to detect and localize the small tumors. The prototype hardware setup uses two Vivaldi antennas, one as transmitter and other as receiver for scanning. The distance between phantom and antennas is kept almost $20 \mathrm{~cm}$. The scattered signals from the high contrast objects emulating the tumor characteristics have been processed through an open-source imaging algorithm, known as MERIT, to construct the image. To get the best results, images are constructed by comparing different algorithms and the varying number of channels. Simulated and measured results are successfully verified for different locations and sizes of conducting objects in a given imaging domain. The proposed system can be used for practical applications in biomedical imaging, especially for the detection of breast cancer at early stages.

Received: August 21, 2020; Accepted: November 24, 2020; Published: December 23, 2020

*Correspondence: Muhammad Zubair, Electrical Engineering Department, Information Technology University, Lahore, Pakistan; Email: muhammad.zubair@itu.edu.pk

Citation: Sami, H., M. Sagheer, M.A. Altaf, J. Iqbal and M. Zubair. 2021. Development of prototype microwave radar-based imaging system for breast cancer detection. Journal of Engineering and Applied Sciences, 39(2): 170-179.

DOI: http://dx.doi.org/10.17582/journal.jeas/39.2.170.179

Keywords: Microwave imaging, Breast cancer detection, Vivaldi antenna, MERIT
\end{abstract}

\section{Introduction}

$\mathrm{B}$ reast cancer can strike at any age and gender, but adult women are more likely to develop it. In 2018, the number of patients who were diagnosed with breast cancer reached 11.6 million worldwide (WCRF International, 2019). The main reason for the high mortality rate is due to false detection of tumors and expensive treatments that people below the poverty line cannot afford. This mortality rate can be reduced to a significant level if the diagnosis of cancer in an early stage is possible. Many current screening techniques such as mammograms are used for the detection of tumors, they use biohazard radiations affecting normal human tissues and, sometimes, may lead to mistreatment. Low ener- gy $\mathrm{X}$-ray radiations are used to identify the malignant tumors that cannot be felt by hands. Mammography is not as effective for the diagnosis of breast cancer in younger females because they have dense tissues. Major drawbacks of mammography include high false negative and false positive rates, use of ionizing radiations, and painful experience (Society). Contrast enhanced digital mammography generates slightly high radiations as compared to mammography. They have a better ability to detect cancer in dense tissues. Ultrasound waves are also used for the detection of tumors, which are low-frequency waves that are not harmful to us, but their precision is low. Ultrasound cannot distinguish between malignant and benign tissues (Kwon et al., 2018). MRI uses magnetic radiations along with radio waves but it is usually not 
recommended due to its false-positive rate, need for experienced radiologists, high cost, time consumption (almost 40-60 minutes are required), and an insufficient number of units (Wang, 2017). Positron emission tomography (PET scan) used radioactive material with glycoprotein or glucose and synthesized in the body. Since tumor cells consume more nutrients than normal cells, they grow faster and when they consume the nutrients, positrons are ejected.These ejected positrons help to make the image of the tumor (Society). PET scans are useful to determine whether cancer has spread to lymph nodes or other parts of the body. However, the drawback of using a PET scan is its low resolution. Because of these issues, there is a significant need for an effective, cost-efficient method for the detection of breast cancer.

In recent years, much research has been done in microwave imaging (MWI) proving it to be successful for non-destructive detection of hidden objects. It is now considered as a precise and harmless method for tumor detection. Besides PET, MRI, and X-ray, MWI uses low power microwaves and their radiation exposure level is under the safe boundaries. There are two factors for the detection of the tumor, one being the size of the tumor and the other being its dielectric properties. MWI is a useful technique because it can differentiate the dielectric properties of normal and tumor tissues. Usually, the dielectric properties of cancer cells are approximately ten times larger than that of normal tissues (Kwon et al., 2018). In 2000, Fear and Stuchly developed an imaging system using an array of antennas for the detection of the artificial spherical tumor (Fear et al., 2003). Small dipole antennas were used as transmitter and receiver. These antennas were low in cost and cover small bandwidth but they were low in efficiency and directivity. In Korea, ETRI developed a system which consists of antennas around cylindrical phantom (Son et al., 2010). A signal in a range of $500 \mathrm{Mhz}$ to $3 \mathrm{GHz}$ is transmitted and its reflections are being recorded. They use Microwave tomography for the construction of Image. At Dartmouth College, they developed a system containing 16 monopole antennas around the phantom (Meaney et al., 2000). A liquid coupling media is used to avoid reflections from the phantom. They used a $0.3-1 \mathrm{GHz}$ frequency band for this operation. In 2016, Goethe University developed a 3D printed phantom that mimics the same electrical properties as the human body (Moll et al., 2016). In another research, Amineh et al. proposed an
Imaging system that uses the TEM horn antenna as transmitter and receiver (Amineh et al., 2011). Due to the directive nature of the horn antenna, it helps in capturing weak signals reflected from the tumor. But it cannot be used for wideband operations. In another study, the Finite Difference Time Domain method is used for tumor detection (Lazaro et al., 2009). To mimic tumor properties, an iron rod bar is used while a mixture of liquids used to present the electrical properties of skin and tissues. In another study at the University of Queensland, researchers proposed an imaging system containing Taper Slot antennas (Mohammed et al., 2013). They used an antenna array around the phantom. An SP6T switch is used for multiplexing. A research group from the California Institute of Technology under the supervision of Dr. Mark Haynes presented a work in which they developed a MWI system that uses numerical techniques for imaging (Haynes et al., 2016). The imaging setup is formed by joining 12 panels to create an imaging cavity. Each panel consists of 3 bow-tie antennas connected parallel with the cavity wall. Due to the low gain of bow-tie antennas, they have less penetration depth which makes the system less efficient for fatty breasts. In 2018, wavelai is developed by a research group (Fasoula et al., 2018). It is a prototype used for clinical trials at Galway University Hospital. They used 18 Vivaldi antennas to capture scattering signals. It takes 10 minutes for a complete scan. In our work, we are using microwave radiography (MWR), which uses reflected waves from the tumor as an input data, and then using the Microwave Radar-based Imaging Toolbox (MERIT), we can construct the image of a tumor at the approximate location. Microwave signal power levels are well under safety levels. Instead of a switch matrix, we are using a rotatory system which is less expensive. A complete scan almost takes 15-20 minutes. So our proposed system is safe from harmful radiation exposure and cost-efficient.

\section{Antenna Design}

For MWI, transmitter and receiver antennas should be directive and wideband.

For the detection of the tumor, the wavelength of the incident wave should be comparable to the size of the tumor. Wideband property of the antenna helps it to detect different sizes of tumor. Due to the directive and wideband property, the Vivaldi antenna has much importance in the domain of Radar-Based 
applications (Fayazi et al., 2012). Vivaldi antenna consists of radiating slots, microstrip feed line, and substrate. It radiates from its exponential flares. The proposed antenna has a size of $10 \mathrm{~cm} \times 10 \mathrm{~cm}(0.67 \lambda$ $\mathrm{x} 0.67 \lambda$ ) where $\lambda$ is the wavelength of the lowest frequency. Firstly, the antenna is designed using conventional equations (Gibson, 1971). For the improvement of gain and directivity antenna is optimized in CST, which yields parameter values given in table 1 . Instead of using a simple microstrip line, it is fed by a radial stub microstrip line. The radial stub microstrip line provides a wideband impedance matching due to its circular shape. The antenna is designed for 2-6 $\mathrm{GHz}$ bandwidth. This operating band is significant because wavelength in this band is comparable to the size of the tumor. $W$ is the total width of antenna, $L$ is the total length of the antenna, $d$ is the cavity diameter, $w$ is the width of transmission line and $l$ is the length of a transmission line. It is simulated on an FR-4 substrate with the dielectric constant of 4.4 and thickness of $1.5 \mathrm{~mm}$. The simulated antenna is shown in Figure 1.

\section{Table 1: Antenna design parameters.}

$\begin{array}{lllll}\mathbf{W} & \mathbf{L} & \mathbf{d} & \mathbf{w} & \mathbf{1} \\ 10 \mathrm{~cm} & 10 \mathrm{~cm} & 0.105 \mathrm{~cm} & 0.304 \mathrm{~cm} & 3.54 \mathrm{~cm}\end{array}$

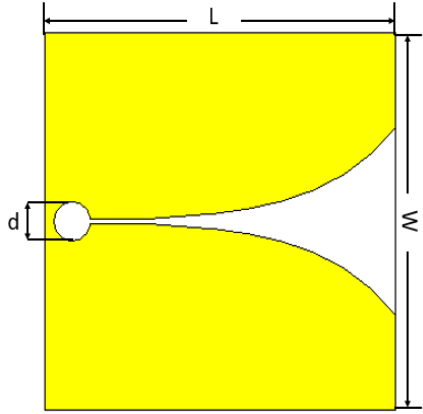

a

Figure 1: Simulated antenna (a) front side (b) backside

\section{Fabricated antenna}

The proposed antenna is fabricated on an FR-4 substrate with a dielectric constant of 4.4. The height of the substrate is $1.5 \mathrm{~mm}$ and the loss tangent of the substrate is 0.001 . It is fed by a $50 \Omega$ coaxial cable through an SMA connector. The fabricated antenna is shown in Figure 2. For Experimental purposes, antenna is fed by National Instruments PXIe 5630 Vector Network Analyzer. This VNA has maximum $5 \mathrm{~mW}(-23 \mathrm{~dB})$ output power (Instruments). Since according to EU and USA maximum Radio waves exposure to the human body is $2 \mathrm{~W} / \mathrm{kg}$ (Madjar, 2016).
So, the exposure of wave in our setup is well under the boundaries. On the other hand, the penetration depth is around $1.5-1.7 \mathrm{~cm}$ at $2.4 \mathrm{GHz}$ depending upon the electrical properties of skin and tissues.
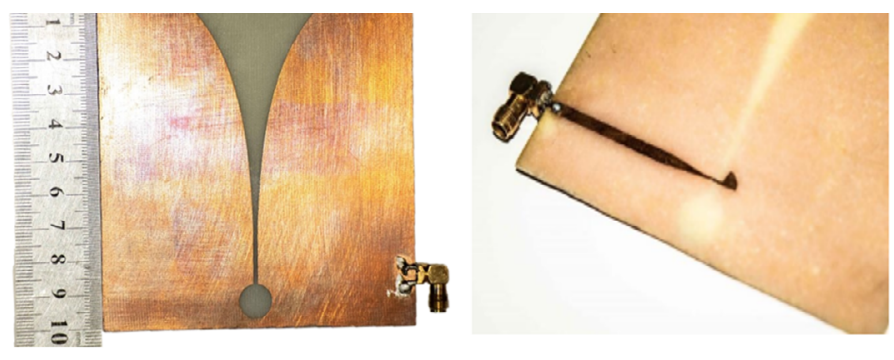
a b

Fig 2. Fabricated antenna (a) Front side (b) Backside

\section{Radiation results}

S11 is measured using National Instruments PXIe 5630 Vector Network Analyzer. The range of the analyzer is $10 \mathrm{MHz}$ to $6 \mathrm{GHz}$. Figure 3 shows the return loss of the simulated and measured antenna. There are some variations in s11 of simulated and measured values due to substrate epsilon difference and thickness of the substrate.

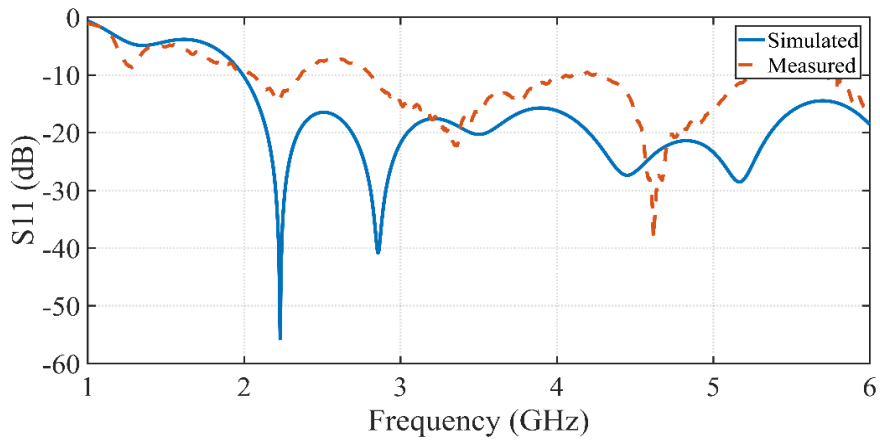

Figure 3: Simulated and measured reflection coefficients of designed Vivaldi antenna

Figure 4 shows the measured and simulated radiation pattern of the proposed antenna. The gain of the antenna is $7.56 \mathrm{dBi}$ and its half-power bandwidth is $60^{\circ}$.

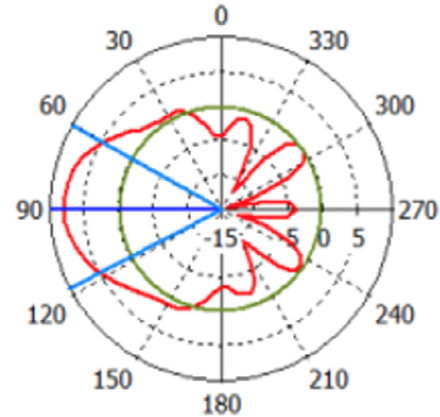

a

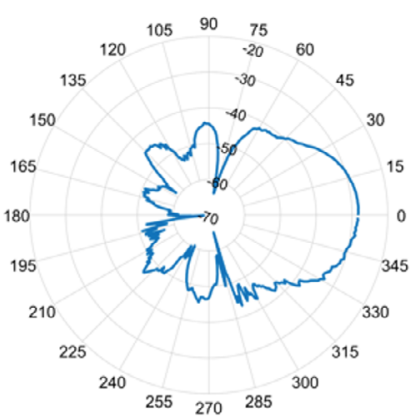

b
Figure 4: Antenna radiation patterns at $4 \mathrm{GHz}(\mathrm{a})$ simulated (b) measured. 


\section{Microwave Imaging}

Microwave radar-based imaging requires the antenna to transmit and receive microwave pulses from different locations around the phantom This process can be done using antenna array (multistate) or single antenna (monostatic). The multistate process involves the multiple antennas that surround the object while monostatic use single antenna which acts as a transmitter as well as a receiver, and this process can be repeated at various locations to collect enough signals. Scattered signals from objects arise due to the high contrast between the electrical properties of normal cells and cancer. These signals contain information about the size and location of the object and are further processed to construct the image.

For MWI, we are using MERIT which is an opensource Microwave Radar-based Imaging Toolbox. MERIT contains a complete package for the microwave radar imaging including the signal processing and parameter search algorithms for dielectric properties (Lavoie et al., 2016; O'loughlin et al., 2017). For imaging, two sets of measured data or scans are required, one taken at a fixed rotational offset from the other to remove the same artifacts and noise from the image. The resolution of the image can be improved by maximum elimination of the noise from the signals and by keeping the minimum distance between the focal points in the imaging domain. Each set of measured data is represented by $\mathrm{L} \times \mathrm{C}$ array, where $\mathrm{L}$ is the length of frequency point in each scan, and $\mathrm{C}$ is the number of channels (O'Loughlin et al., 2012). It also requires data consist of all the frequency points used for scan files, the location of the antennas, and the combination of channels.

Two methods will be tested using MERIT to find an effective way to construct the image of the tumors. The first method is the varying position of the transmitter, in which the position of transmitter and receiving antennas varies. The transmitting antenna changes its position to complete $360^{\circ}$ rotation around the phantom. The total channel number can be calculated by ${ }^{\mathrm{n}} \mathrm{C}_{2}$ where $\mathrm{n}$ is the total number of the antennas. The other method is the fixed transmitter method in which single transmitter antenna is used at fixed position and therefore less time consuming than the varying transmitter method. The comparison between both methods is done by using three algorithms Delay and Sum (DAS), Delay Multi- ply and Sum (DMAS) and Coarse Delay and Sum (CDAS). In DAS, scattered signals are time-shifted corresponding to their time delays. These times shifted scattered signals are summed from each channel and the energy is computed at each focal point (in imaging domain). The energy from summed signals is used to construct the profile of hemispherical imaging domain and highlights the point with the high contrast of dielectric properties (O'Loughlin et al., 2012). DMAS is the extended version of DAS. It introduces pairing multiplication that increases the signals from $\mathrm{M}$ to ${ }^{\mathrm{M}} \mathrm{C}_{2}$ (Lim et al., 2008). Pairing multiplication is carried out before the signals are summed at each focal point. DMAS ensures additional clutter rejection with an increase in the sample size which helps to improve the tumor detection capabilities. CDAS also enhances DAS by assigning the quality factor at each focal point. It suppresses the points with a lower degree of coherence and eventually leads to clutter rejection.

\section{Hardware Setup}

Two Vivaldi antennas are used in this setup. Rods (selected with the material that has no of effect on the conductance with the antenna) are used to attach antennas with motors. One end of the rod is attached to the antenna and the other end of the rode is attached with stepper motor (NEMA 23, 2.2A). Both motors are driven by their drivers (Tb6560, 3A, 12-24V). Arduino Uno is used as a controller to rotate the antennas in the circle around the phantom to capture the scattering parameters using VNA (PXIe-5630, $10 \mathrm{MHz}$ to $6 \mathrm{GHz}$ ). Since switching matrix can also be used to feed antenna array around phantom but it is not a cost-efficient solution. To avoid the switching matrix, only two antennas are used with the rotatory system. RX and TX antennas can be rotated to capture signals from other positions around phantom (Mohammed, 2014).

\section{Implementation scheme}

In our setup, two scans around the phantom are used to form an image. In the first scan, the transmitter is located at $\varphi$ angle and the receiver is located at $\theta$ angle. TX angle $\theta$ will start from $0^{\circ}$. Rx can rotate around the phantom with the difference of step angle $\Delta \varphi$. Where $\Delta \varphi$ can be calculated as $\Delta \varphi=\frac{360}{p}$. Here $p$ is the number of positions around the phantom where you want to capture signals. Here $\Delta \varphi$ is the desired step angle to measure the scattering parameters for the first rota- 
tion of the receiver. We can also select $\underline{\varphi}$ according to our measurement domain. For the other rotations of the receiver, the initial angles of the transmitter and receiver are set by adding the offset of the step angle in their previous initial angles. For the second scan, we only need to add an offset initially in the angles of the transmitter and receiver by half of the desired step angle. Now $\theta$ will start from (0+offset) degree. Note smaller step angle means a greater number of positions around phantom, which improves the resolution of the image. For example, we can capture signals around phantom at 8 positions when the step angle is $45^{\circ}$. When the step angle is $30^{\circ}$, there are 12 positions from where we can collect signals. Figure 5 shows the implementation of the hardware setup with two antennas. Note that the phantom will be scanned in the near field.

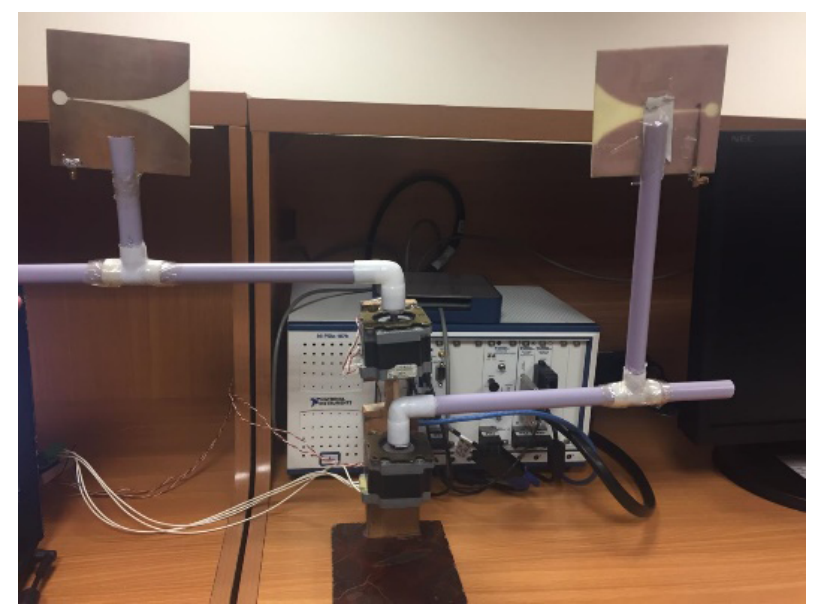

Figure 5: Implemented hardware prototype.

Communication of labview with Arduino and VNA The flow chart of measuring the scattering parameters by using the communication of labview with $\mathrm{Ar}-$ duino and VNA is shown in Fig 6.

In the first step, the desired step angle is given by the LabView as an input to Arduino to calculate the combinations and to rotate the receiver according to the step angle. Then, a command is sent from Arduino to LabView for creating a CSV file and then LabView sends a command to VNA for measuring the scattering parameters, then these scattering parameters are saved in CSV file. After saving the scattering parameters, a command is sent to Arduino by LabView to rotate the receiver again according to the step angle, and in each iteration of the Arduino, the combinations are calculated and compared to the desired combinations. Then a command is sent to LabView to again create a file and save the scattering parameters into the file.
The whole procedure is continued until the receiver reaches the maximum position (i.e. $<360^{\circ}$ ). The total CSV files gathered at the end of the procedure should be equal to the total desired combinations. These files are then used in Matlab to construct an image. Figure 7 shows the control flow diagram of the complete control process and LabVIEW front panel control.

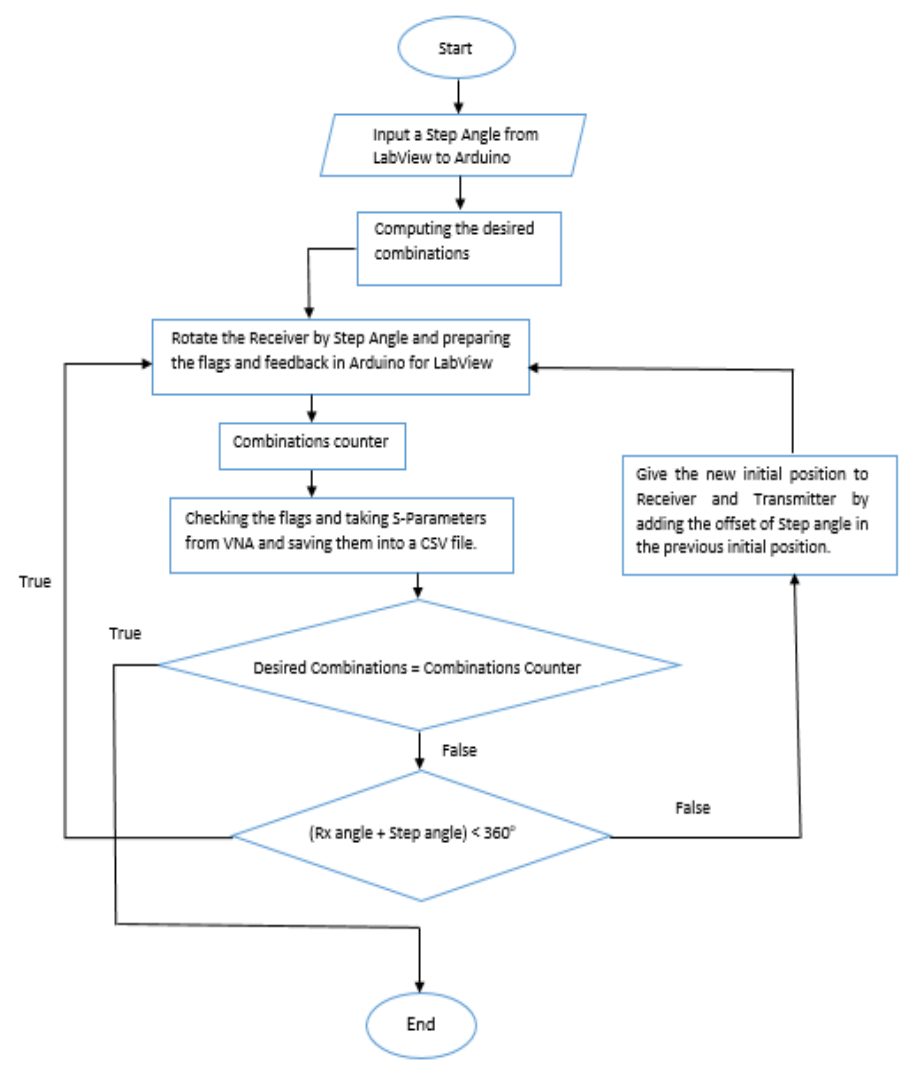

Figure 6: Flow chart for control of measurement setup.

\section{Results and Discussions}

To test the detection abilities of a designed microwave imaging system, we compare fixed and varying transmitter method. A cylindrical rod with different sizes is selected as a testing object and the position of the cylindrical rod is changed in different cases to verify the exact location of the object in the imaging domain. The comparison between the fixed and varying transmitter method will be tested on the following parameters:

1. The number of channels will remain the same for both methods.

2. For imaging, different algorithms such as Delay and Sum (DAS), Delay Multiply and Sum (DMAS) and Coarse Delay and Sum (CDAS) will be tested.

Starting from four antennas, which give six channels 
by using ${ }^{\mathrm{n}} \mathrm{C}_{2}$, no object was detected using any algo- Figure 8: Object detection (a-c) varying transmitter rithm. By increasing the number of channels to 28, method ( $\mathrm{d}-\mathrm{f}$ ) fixed transmitter method. Off all the the object was successfully identified using DMAS. algorithms used, DMAS is shown to have acceptable All these images are added after the normalization. performance.

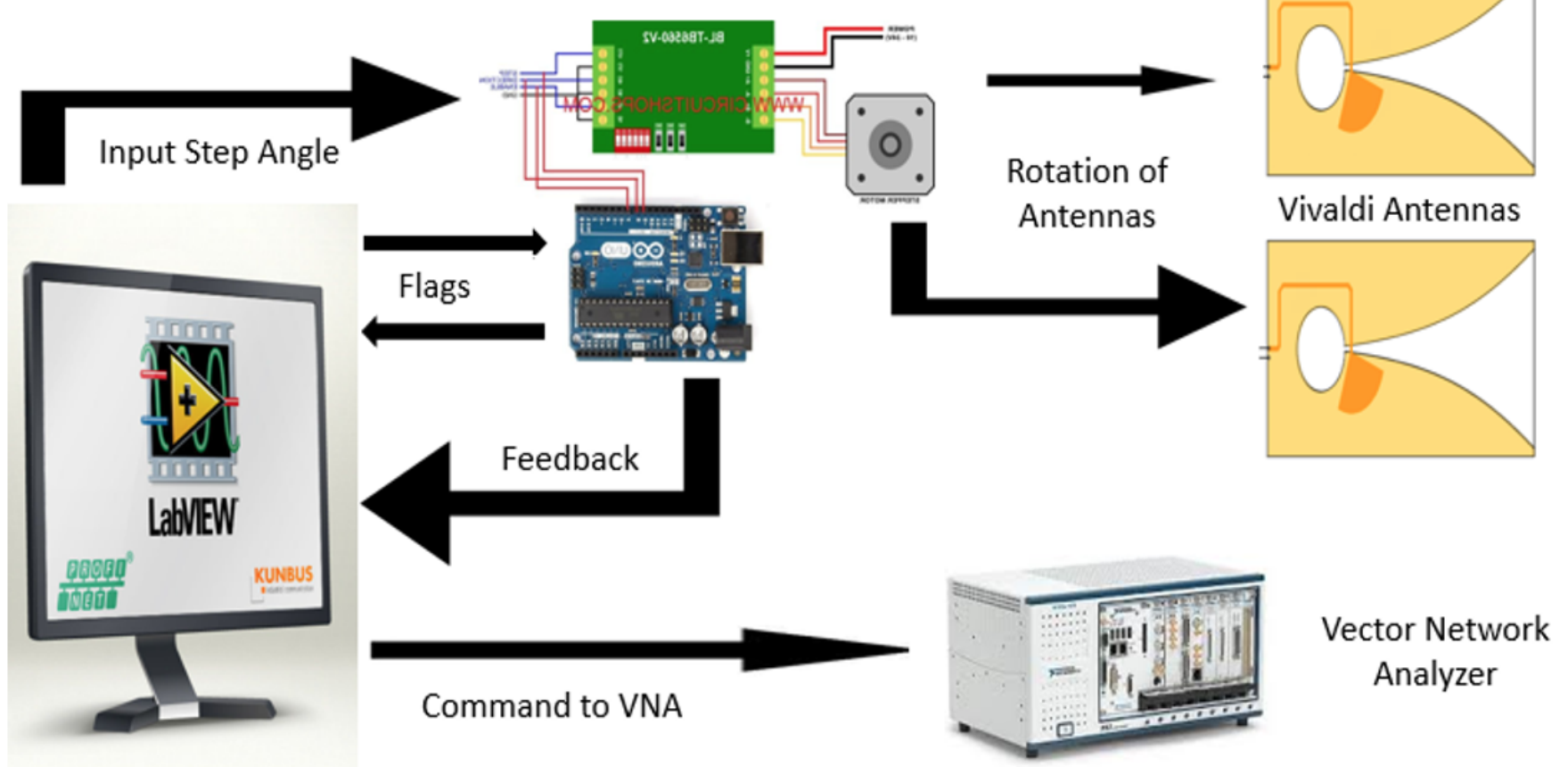

(a)

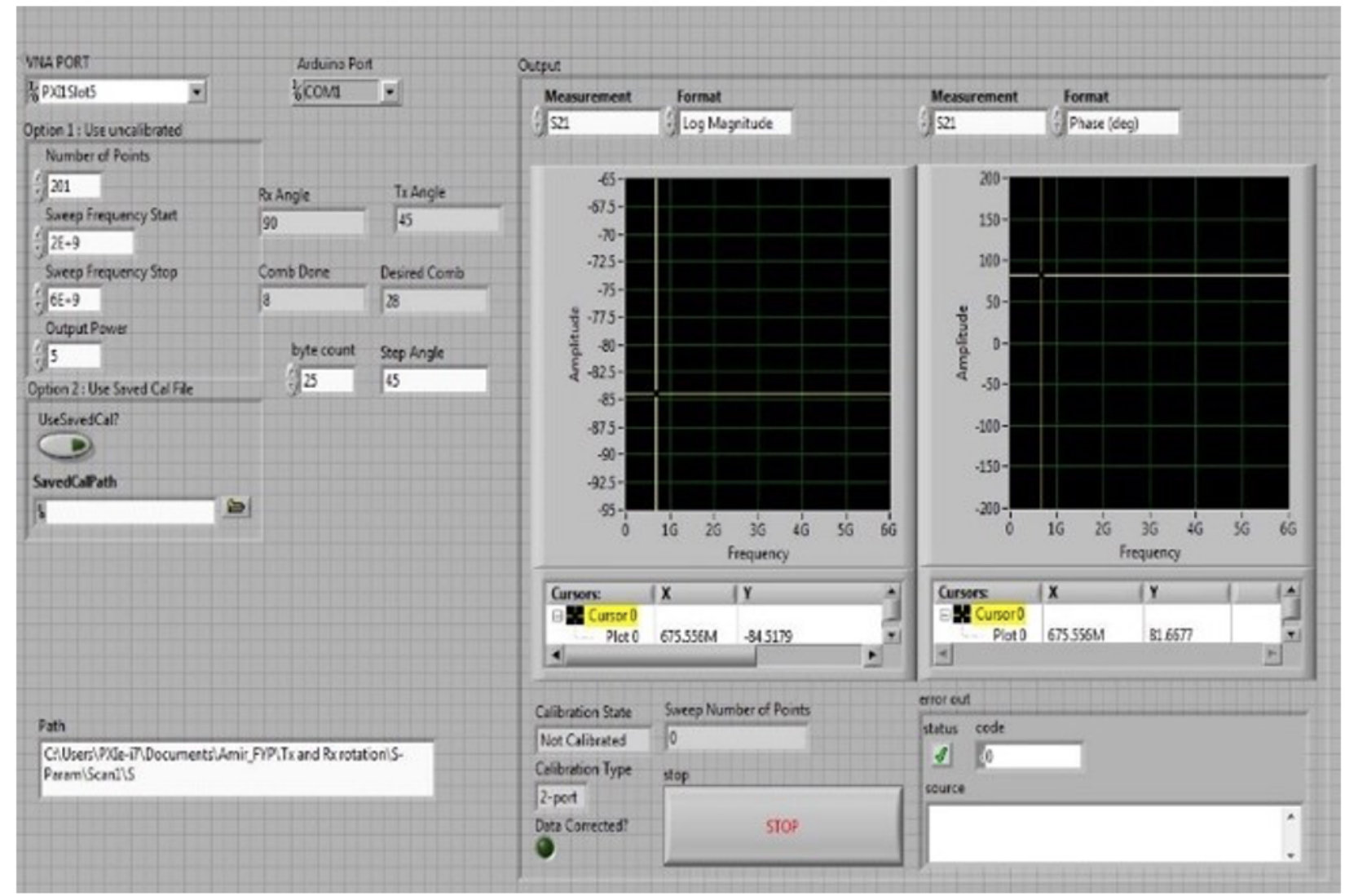

(b)

Figure 7: Data acquisition process (a) control flow diagram (b) implemented labview front panel December 2020 | Volume 39 | Issue 2 | Page $175 \quad \begin{array}{r}\text { Links } \\ 0.000\end{array}$ 


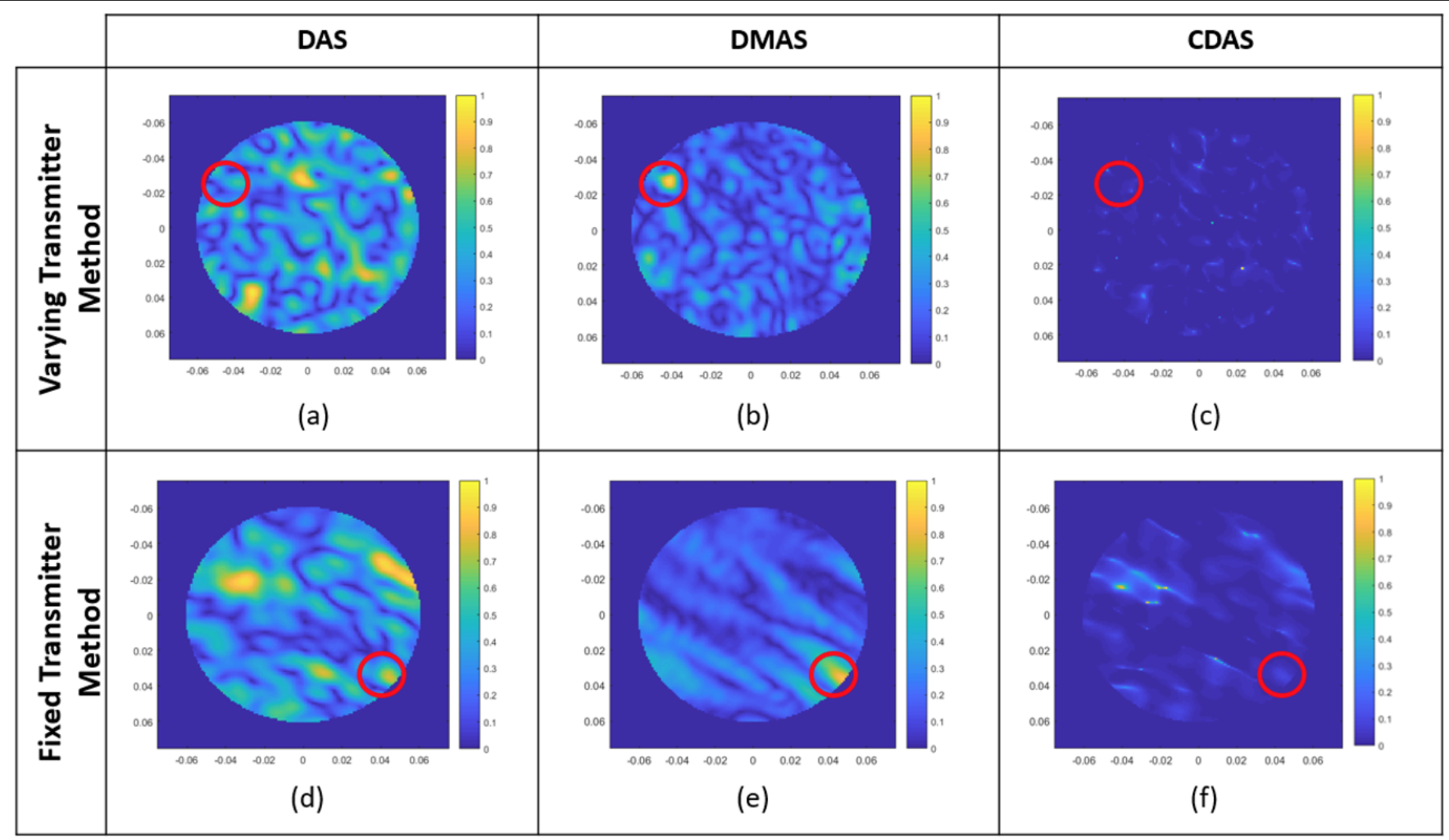

Figure 8: Object detection (a-c) varying transmitter method $(d-f)$ fixed transmitter method. Off all the algorithms used, DMAS is shown to have acceptable performance.

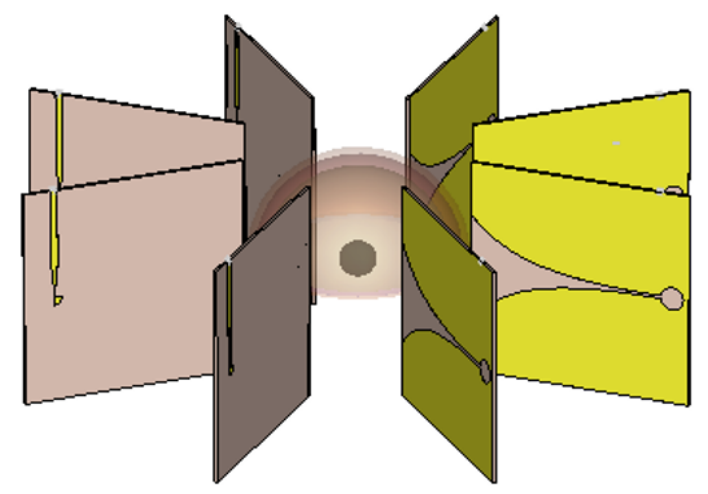

(a)

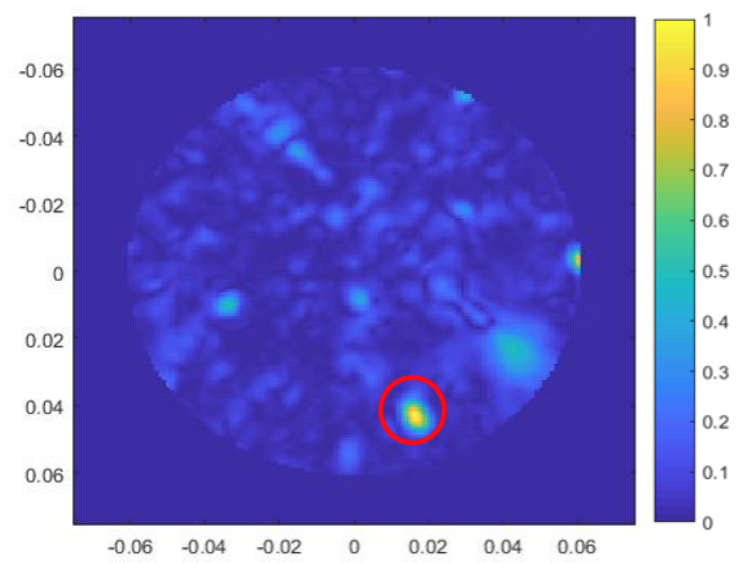

(c)

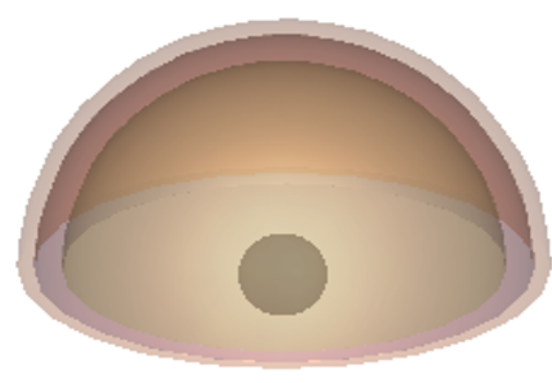

(b)

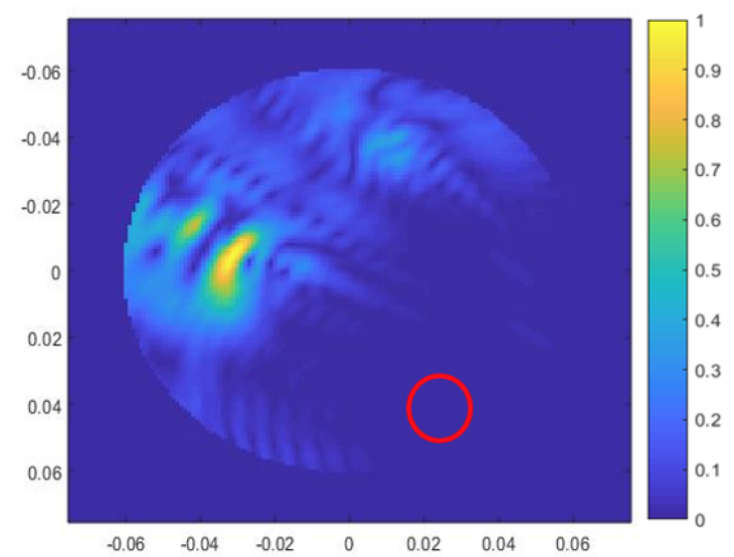

(d)

Figure 9: CST simulation (a) designed Vivaldi antennas around breast phantom (b) tumor placed inside breast phantom (c) varying transmitter method (d) fixed transmitter method. It is shown that the varying transmitter method has acdeoptable performance. 


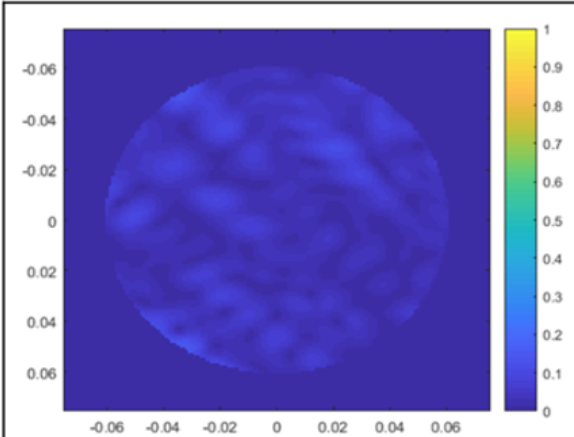

(a)

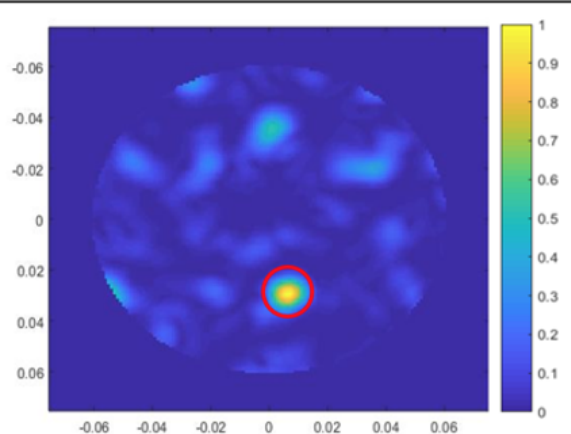

(b)

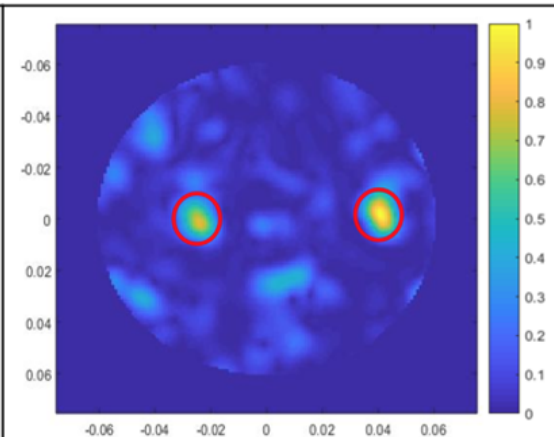

(c)

Figure 10: (a) Comparison with reference (without object) (b) Object position changes (c) Two objects

In Figure 8, a single object is placed inside the imaging domain and the red circle represents its actual position. In the above row of Figure 8, the detection is done using varying transmitter method. DAS and CDAS algorithm failed to detect the object in this case but DMAS which have better clutter rejection property detects the object. After that, the position of the object is changed, and scattering parameters are extracted using the fixed transmitter method. DAS shows the object is detected in various positions which didn't depict the actual case. DMAS in this case also successfully detects the tumor while CDAS detects nothing. The resolution of image can be improve by better removal of noise from the signals. After comparing both methods using measured data, these methods are verified in CST software.

To mimic the properties of actual tissues in CST, the breast phantom was designed using electrical properties and conductivity of the skin, fats, glandular tissues, and tumor for 2-6 GHz (Oliveira et al., 2018). The size of the tumor was set to 5-mm. Figure $9(a-b)$ shows Vivaldi antennas around the breast phantom and the tumor is placed inside the phantom. Figure 9(c) represents image constructed using varying transmitter method, which locates the tumor while the fixed transmitter method fail to locate the tumor as shown in Figure 9(d).

After verification from CST, various experiments were performed using the hardware setup to further verify the varying transmitter method. Using the setup of object detection in Figure 8 (b) as a reference, more experiments were performed. At first, imaging was performed without any object present in the imaging domain to check the specificity of the proposed MWI. The constructed image shows that no object is detected as seen in Figure 10 (a). After that, the position of the object is changed from the reference position and the constructed image also shows the object position is changed which is shown in Figure 10 (b). Then two objects of different sizes were placed in the imaging domain and the microwave imaging successfully locates these two objects (Figure 10 (c)). After analyzing all the results we can conclude that a minimum of 28 channels is required to localize the tumor. Different algorithms were tested including DAS, DMAS, and CDAS from which only DMAS prove to be successful at locating the tumor.

\section{Conclusions}

In this paper, a complete microwave imaging system with a compact size Vivaldi antenna is proposed. The Vivaldi antenna has the bandwidth of $2-6 \mathrm{GHz}$ and the highest gain of $7.56 \mathrm{dBi}$ to overcome the body losses. A state of the art hardware setup is designed to measure scattering parameters. Stepper motors are used to rotate the antennas around the phantom which are controlled by Arduino. The imaging algorithm (MERIT) uses these scattering parameters and removes the noise from the signals and constructs the image of the tumor with a minimum of 28 channels and using the DMAS algorithm. In particular, varying transmitter method outperforms the fixed transmitter method as it allows the transmitter and receiver to fully scan the phantom by changing their positions.

\section{Acknowldgement}

This publication is based on the research that has been supported by the Technology Development Fund (TDF), Higher Education Commission of Pakistan.

\section{Novelty Statement}

This works presents a prototype of low-cost, 
easy-to-implement microwave imaging system for pre-screening of breast cancer.

\section{Author's Contribution}

Humza Sami: Worked on development and testing of antennas and contributed to writing of manuscript. Mahnoor Sagheer: Worked on running imaging algorithm on the measured data and contributed to writing of manuscript.

Muhammad Amir Altaf: Developed the LabView framework for automation of overall measurement setup.

Javaid Iqbal: Advised on measurement procedure and contributed to writing of manuscript.

Muhammad Zubair: Conceived the initial idea, supervised the hardware implementation, and development of software part. Also contributed to the writing of manuscript.

\section{Conflict of interest}

The authors have declared no conflict of interest.

\section{References}

Amineh, R. K., Ravan, M., Trehan, A., and Nikolova, N.K. 2011. Near-field microwave imaging based on aperture raster scanning With TEM horn antennas. IEEE Transactions on Antennas and Propagation, 928-940.

Fasoula, A., Duchesne, L., Cano, J. D., Lawrence, P., Robin, G., and Bernard, J.-G. 2018. On-site validation of a microwave breast imaging system, before first patient study. Diagnostics(Besel), 8(3), 53.

Fayazi, S. S., Lui, H.-S., and Yang, J. 2012. Microwave imaging of near-field object using ultra-wideband synthetic aperture radar algorithm. IEEE International Symposium on Antennas and Propagation, 1-2.

Fear, E. C., Meaney, P.M., and Stuchly, M.A. 2003. Microwaves for breast cancer detection? IEEE Potentials, 12-18.

Gibson, P. 1971. The Vivaldi Aerial. European Microwave Conference, 101-105.

H. M. Madjar. 2016. Human radio frequency exposure limits: An update of reference levels in Europe, USA, Canada, China, Japan and Korea. 467-473.

Haynes, M., Stang, J., and Moghaddam, M. 2012. Microwave breast imaging system prototype with integrated numerical characterization. Internation Journal of Biomedical Imaging, 20122), 706365.

Instruments, N. (n.d.). PXIe-5630,” PXIe-5630. Retrieved from https://www.ni.com/enlb/ support/model.pxie-5630.html

Kwon, S., and Lee, S.2018. Corrigendum to "Recent advances in microwave imaging for breast cancer. International Journal of Biomedical Imaging.

Lavoie, B. R., Okoniewski, M., and Fear, E. C. 2016. Estimating the effective permittivity for reconstructing accurate microwave-radar images. Plos One, 11(9), 1-25.

Lazaro, A., Girbau, D., and Villarino, A. R. 2009. Simulated and experimental investigation of microwave imaging using UWB. Progress in Electromagnetics Research, 23, 263-280.

Lim, H. B., Nhung, N. T., Li, E.-P., and Thang, N. D. 2008. Confocal Microwave Imaging for Breast Cancer Detection: Delay-Multiply-andSum Image Reconstruction Algorithm. IEEE Transactions on Biomedical Engineering, 55(6), 1697.

Meaney, P. M., Fanning, M. W., Li, D., Poplack, S. P., and Paulsen, K. D. 2000. A clinical prototype for active microwave imaging of the breast. IEEE Transactions on Microwave Theory and Techniques, 1841-1853.

Mohammed, B. 2014. Design and implementation of microwave imaging systems for medical applications. School of Information Technology and Electrical Engineering, The University of Queensland.

Mohammed, B. J., Abbosh, A. M., and Sharpe, P. 2013. Planar array of corrugated tapered slot antennas for ultrawideband biomedical microwave imaging system. International Journal of RF and Microwave, 23, 59-66.

Moll, J. M., Wörtge, D., Byrne, D., Klemm, M., and Krozer, V. 2016. Experimental phantom for contrast enhanced microwave breast cancer detection based on 3D printing technology. European Conference on Antennas, 1-4.

Oliveira, B. L., O'Loughlin, D., O'Halloran, M., Porter, E., Glavin, M., and Jones, E. 2018. Microwave Breast Imaging: Experimental tumour phantoms for the evaluation of new breast cancer diagnosis systems. Biomedical Physics and Engineering Express, 42), 025036. O'Loughlin, D., Elahi, M. A., Porter, E., Shahzad, 
A., Oliveira, B. L., Glavin, M., and Jones, E. 2012. Open-source software for microwave radar-based image reconstruction. 12th European Conference on on Antennas and Propagation, 1-4.

O'loughlin, D., Krewer, F., Glavin, M.,Jones, E., and O'halloran, M. 2017. Focal quality metrics for the objective evaluation of confocal microwave images. International Journal of Microwave and Wireless Technologies, 9(7), 1365-1372.

Society, A. C. (n.d.). Imaging Test To Find Out If Breast Cancer Has Spread. Retrieved from https://www.cancer.org/cancer/breast-cancer/ screening-tests-and-early-detection/tests-tofind-out-if-breast-cancer has-spread.html

Son, S. H., Simonov, N., Kim, H. J., Lee, J. M., and Jeon, S. I. 2010. Preclinical prototype development of a microwave tomography system for breast cancer detection. Etri Journal, 901-910.

Wang, L. 2017. Early Diagnosis of Breast Cancer. Sensors, 17(7), 1572.

WCRF International. 2019. Breast cancer statistics. Retrieved from https://www.wcrf. org/dietandcancer/cancer-trends/breastcancerstatistics. 